\title{
INNER TABLEAU TRANSLATION PROPERTY OF THE WEAK ORDER AND RELATED RESULTS
}

\author{
MÜGE TAŞKIN \\ (Communicated by Jim Haglund)
}

\begin{abstract}
Let $S Y T_{n}$ be the set of all standard Young tableaux with $n$ cells and $\leq_{\text {weak }}$ be Melnikov's weak order on $S Y T_{n}$. The aim of this paper is to introduce a conjecture on the weak order, named the inner tableau translation property. We prove the conjecture for some special cases and discuss its significance.
\end{abstract}

\section{INTRODUCTION}

The weak order on $S Y T_{n}$ is introduced by Melnikov and well studied in [15. [16. 17] due to its strong connections to Kazhdan-Lusztig and geometric order on standard Young tableaux. Roughly speaking,

- the weak order is induced from the weak Bruhat order on the symmetric group $S_{n}$ via the Robinson-Schensted-Knuth (RSK) insertion map,

- the KL order is induced by the Kazhdan-Lusztig preorder on $S_{n}$ arising in the theory of Kazhdan-Lusztig (right) cells, and

- the geometric order describes inclusions of certain algebraic varieties indexed by tableaux (orbital varieties).

All of these orders on $S Y T_{n}$ coincide for $n \leq 5$, as depicted in Figure 1. In general we have

$$
\text { weak order } \subsetneq \text { Kazhdan-Lusztig (KL) order } \subseteq \text { geometric order, }
$$

where $P \subset Q$ means that $u \leq v$ in $P$ implies $u \leq v$ in $Q$, in which case we say that $Q$ is stronger than $P$ (or $P$ is weaker than $Q$ ).

Due to its definition, which uses just the combinatorics of tableaux such as the RSK insertion and the weak Bruhat order on the symmetric group, the weak order has an important place among these orders. On the other hand, the only justification for its well-definedness relies on the Kazhdan-Lusztig (KL) or the geometric order through the above inclusion (1.1); i.e., there is no self-contained combinatorial proof of this basic fact.

Our aim here is to draw attention to a conjecture on the inner tableau translation property of the weak order. This property is first mentioned in 25] and it is known to be true for Kazhdan-Lusztig and geometric orders. Its importance on the weak order, as is shown in Section 3 , relies on the fact that it provides a self-contained proof for the well-definedness of this order.

Received by the editors February 8, 2011 and, in revised form, July 25, 2011.

2010 Mathematics Subject Classification. Primary 05E10, 20 C30.

(C)2012 American Mathematical Society Reverts to public domain 28 years from publication 
Conjecture 1.1. Let $R, \tilde{R} \in S Y T_{k}$ be two tableaux having the same shape. For $S, T \in S Y T_{n}$ having the same inner tableaux $R$, let $\tilde{S}$ and $\tilde{T}$ be the tableaux which is obtained by replacing $R$ with $\tilde{R}$ in $S$ and $T$ respectively. Then

$$
S \leq_{\text {weak }} T \text { if and only if } \tilde{S} \leq_{\text {weak }} \tilde{T} \text {. }
$$

In the following we first provide the definitions and related background for the weak order on $S Y T_{n}$. In the third section, by assuming the conjecture, we provide a self-contained proof for the well-definedness of the weak order and we also discuss how this conjecture plays a specific role in the theory of Poirier and Reutenauer Hopf algebra on standard Young tableaux. In the last section, we prove the conjecture for the cases when the inner tableau $R$ has a hook shape or a shape which consists of two rows or two columns. Our method strongly depends on the dual Knuth relations and has certain similarities with the one used in the work of Reifegerste [20].

\section{RELATED BACKGROUND}

2.1. Definition of the weak order. Recall that a sequence $\lambda=\left(\lambda_{1}, \ldots, \lambda_{k}\right)$ is a partition of $n, \lambda \vdash n$, if $\sum_{i=1}^{k} \lambda_{i}=n$ and $\lambda_{i} \geq \lambda_{i+1}>0$, where its representation as a Young diagram consists of left justified arrows of boxes such that the $i$-th row has $\lambda_{i}$ boxes. A partition $\lambda=\left(\lambda_{1}, \ldots, \lambda_{k}\right)$ can also be seen as a set of integer pairs $(i, j)$ such that $1 \leq i \leq k$ and $1 \leq j \leq \lambda_{i}$. For example,

$$
\lambda=(2,1)=\square=\{(1,1),(1,2),(2,1)\} .
$$

For two partitions $\lambda$ and $\mu$ satisfying $\mu \subset \lambda$, the diagram which is defined as $\lambda / \mu=\{c: c \in \lambda, c \notin \mu\}$ is call a skew diagram. Now a skew diagram is called normal if $\mu=\varnothing$. A partial skew (normal) tableau of shape $\lambda / \mu(\lambda)$ is obtained by labeling $\lambda / \mu(\lambda)$ by distinct integers such that the labels increase from left to right and from top to bottom. A standard skew (Young) tableau of shape $\lambda / \mu(\lambda)$ is a partial skew (normal) tableau whose labels are $\{1,2, \ldots, n\}$. If $T$ is such a tableau, then we set

$$
\operatorname{shape}(T)=\lambda / \mu(\lambda) .
$$

The definition of the weak order uses well-known Robinson-Schensted-Knuth $(R S K)$ correspondence which bijectively assigns every permutation $w \in S_{n}$ to a pair of tableaux $(I(v), R(w)) \in S Y T_{n} \times S Y T_{n}$, having the same shape, where $I(w)$ and $R(w)$ are called the insertion and recording tableau of $w$ respectively.

Let us explain these algorithms briefly. Let $I_{i-1}$ and $R_{i-1}$ be the tableaux obtained by inserting and respectively recording the first $i$ indices of $w=w_{1} \ldots w_{n}$. Now $I_{i}$ is obtained by inserting $w_{i}$ into $I_{i-1}$, denoted as

$$
I_{i}=\left(I_{i-1}\right)^{\downarrow w_{i}}
$$

in the following manner: if $w_{i}$ is greater then the rightmost number on the first row of $I_{i-1}$, then $w_{i}$ is concatenated to the right side of this row. Otherwise $w_{i}$ replaces the smallest number, say $a$, among all numbers in the first row greater than $w_{i}$ and this time $a$ is inserted in the next row. Observe that after finitely many steps the insertion algorithm terminates with a new added (corner) cell. The resulting tableau is then called $I_{i}$. On the other hand, the recording tableau $R_{i}$ is obtained 
from $R_{i-1}$ by labeling this new corner cell by $i$. We illustrate these algorithms with the following example.

Example 2.1. Let $w=52413$. Then,

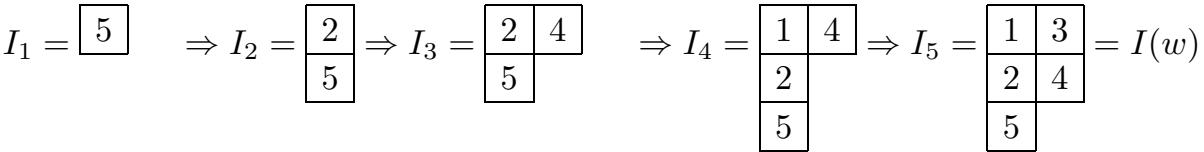

$$
\begin{aligned}
& R_{1}=1 \Rightarrow R_{2}=\begin{array}{|l|l|}
\hline 1 \\
\hline 2
\end{array} \Rightarrow R_{3}=\begin{array}{|l|l}
\hline 1 & 3 \\
\hline 2 & \Rightarrow
\end{array} \Rightarrow R_{4}=\begin{array}{|l|l|}
\hline 1 & 3 \\
\hline 2 & \Rightarrow
\end{array} \Rightarrow R_{5}=\begin{array}{|l|l|}
\hline 1 & 3 \\
\hline 2 & 5
\end{array}=R(w)
\end{aligned}
$$

An equivalence relation introduced by Knuth [10] on $S_{n}$ plays a crucial role in this correspondence.

Definition 2.2. We say that $u, w \in S_{n}$ differ by a single Knuth relation if

$$
u=u_{1} \ldots u_{i-1} u_{i} u_{i+1} u_{i+2} \ldots u_{n} \text { and } w=u_{1} \ldots u_{i-1} u_{i+1} u_{i} u_{i+2} \ldots u_{n},
$$

where either $u_{i-1}$ or $u_{i+2}$ lies between $u_{i}$ and $u_{i+1}$. Two permutations are called Knuth equivalent, written $u \underset{K}{\sim} w$, if one of them can be obtained from the other by applying a sequence of Knuth relations.

The following result is also due to Knuth [10].

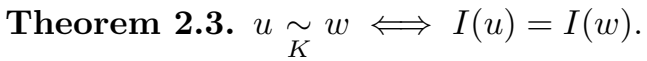

By the last result we now parameterize Knuth equivalence classes by the standard Young tableaux. Namely

$$
\left\{\mathcal{Y}_{T}\right\}_{T \in S Y T_{n}}
$$

denotes the set of Knuth equivalence classes in $S_{n}$.

Schützenberger's backward and forward jeu de taquin slides [24] are one of the most important combinatorial operations on tableaux that we apply often. Skipping the definition (see [21] for details), we illustrate below the backward jeu de taquin slides through the cell indicated by a dot.

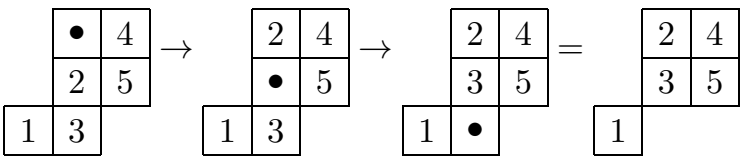

The other main ingredient of the weak order is the (right) weak Bruhat order, $\leq_{\text {weak }}$, on $S_{n}$. Recall that this order is obtained by taking the transitive closure of the following relation:

$$
u \leq_{\text {weak }} w \text { if } w=u \cdot s_{i} \text { and } \operatorname{length}(w)=\operatorname{length}(u)+1,
$$

where $s_{i}$ denotes the adjacent transposition $(i, i+1)$ and length $(w)$ measures the size of a reduced word of $w$. The weak order has an alternative characterization 3 , Prop. 3.1] in terms of (left) inversion sets, namely

$$
u \leq_{\text {weak }} w \text { if and only if } \operatorname{Inv}_{L}(u) \subset \operatorname{Inv}_{L}(w),
$$

where $\operatorname{Inv}_{L}(u):=\left\{(i, j): 1 \leq i<j \leq n\right.$ and $\left.u^{-1}(i)>u^{-1}(j)\right\}$. 
Now the definition of the weak order, which was first introduced by Melnikov [15] under the name induced Duflo order, is the following:

Definition 2.4. The weak order $\left(S Y T_{n}, \leq_{\text {weak }}\right)$ is the partial order induced by taking the transitive closure of the following relation:

$$
S \leq_{\text {weak }} T \text { if there exist } \sigma \in \mathcal{Y}_{S}, \tau \in \mathcal{Y}_{T} \text { such that } \sigma \leq_{\text {weak }} \tau .
$$

The necessity of taking the transitive closure in the definition of the weak order is illustrated by the following example (cf. Melnikov [15, Example 4.3.1]).

$$
\begin{aligned}
& \text { Example 2.5. Let } R=\begin{array}{|l|l|l}
\hline 1 & 2 & 5 \\
\hline 3 & 4 &
\end{array}, S=\begin{array}{|l|l|l|}
\hline 1 & 4 & 5 \\
\hline 2 &
\end{array}, T=\begin{array}{|l|l|}
\hline 1 & 4 \\
\hline 2 & 5 \\
\hline 3
\end{array} \quad \text { with } \\
& \mathcal{Y}_{R}=\{31425,34125,31452,34152,34512\} \text {, } \\
& \mathcal{Y}_{S}=\{32145,32415,32451,34215,34251,34521\} \text {, } \\
& \mathcal{Y}_{T}=\{32154,32514,35214,32541,35241\} \text {. }
\end{aligned}
$$

Here $R<_{\text {weak }} S$ since $34125<_{\text {weak }} 34215$, and $S<_{\text {weak }} T$ since $32145<_{\text {weak }} 32154$. Therefore $R<_{\text {weak }} T$. On the other hand, for every $\rho \in \mathcal{Y}_{R}$ and for every $\tau \in \mathcal{Y}_{T}$ we have $(2,4) \in \operatorname{Inv}_{L}(\rho)$ but $(2,4) \notin \operatorname{Inv}_{L}(\tau)$.

\subsection{Some basic properties of the weak order.}

Definition 2.6. Let $[i, j]$ be a segment in $[1, n]$. Then let

i) $u_{[i, j]}$ be the subword $u \in S_{n}$ consisting of the numbers in the segments $[i, j]$ and $\operatorname{std}\left(u_{[i, j]}\right) \in S_{j-i+1}$ be the permutation obtained by subtracting $i-1$ from each number of $u_{[i, j]}$;

ii) $S_{[i, j]}$ be the normal shape tableau obtained by applying Schützenberger's backward jeu-de-taquin slides on the (partial skew) subtableau of $S$ which consists of the numbers in segments $[i, j]$. Now let

$$
\operatorname{std}\left(S_{[i, j]}\right) \in S Y T_{j-i+1}
$$

be the tableau obtained by subtracting $i-1$ from each number in $S_{[i, j]}$.

In fact $\operatorname{Inv}_{L}(u) \subset \operatorname{Inv}_{L}(w)$ gives $\operatorname{Inv}_{L}\left(u_{[i, j]}\right) \subset \operatorname{Inv}_{L}\left(w_{[i, j]}\right)$ for all $1 \leq i<j \leq n$ and hence

$$
u \leq_{\text {weak }} w \text { implies } \operatorname{std}\left(u_{[i, j]}\right) \leq_{\text {weak }} \operatorname{std}\left(w_{[i, j]}\right) \text { for all } 1 \leq i<j \leq n .
$$

The following basic fact about $R S K$, Knuth equivalence, and jeu-de-taquin are essentially due to Knuth and Schützenberger; see Knuth [9, Section 5.1.4] for detailed explanations.

Lemma 2.7. Given $u \in S_{n}$, let $I(u)$ be the insertion tableau of $u$. Then for $1 \leq i<j \leq n$,

$$
\operatorname{std}\left(I(u)_{[i, j]}\right)=I\left(\operatorname{std}\left(u_{[i, j]}\right)\right) .
$$

Therefore we have the following:

Lemma 2.8. The weak order restricts to segments; i.e.,

$$
S \leq T \text { implies } \operatorname{std}\left(S_{[i, j]}\right) \leq \operatorname{std}\left(T_{[i, j]}\right) \text { for all } 1 \leq i<j \leq n .
$$




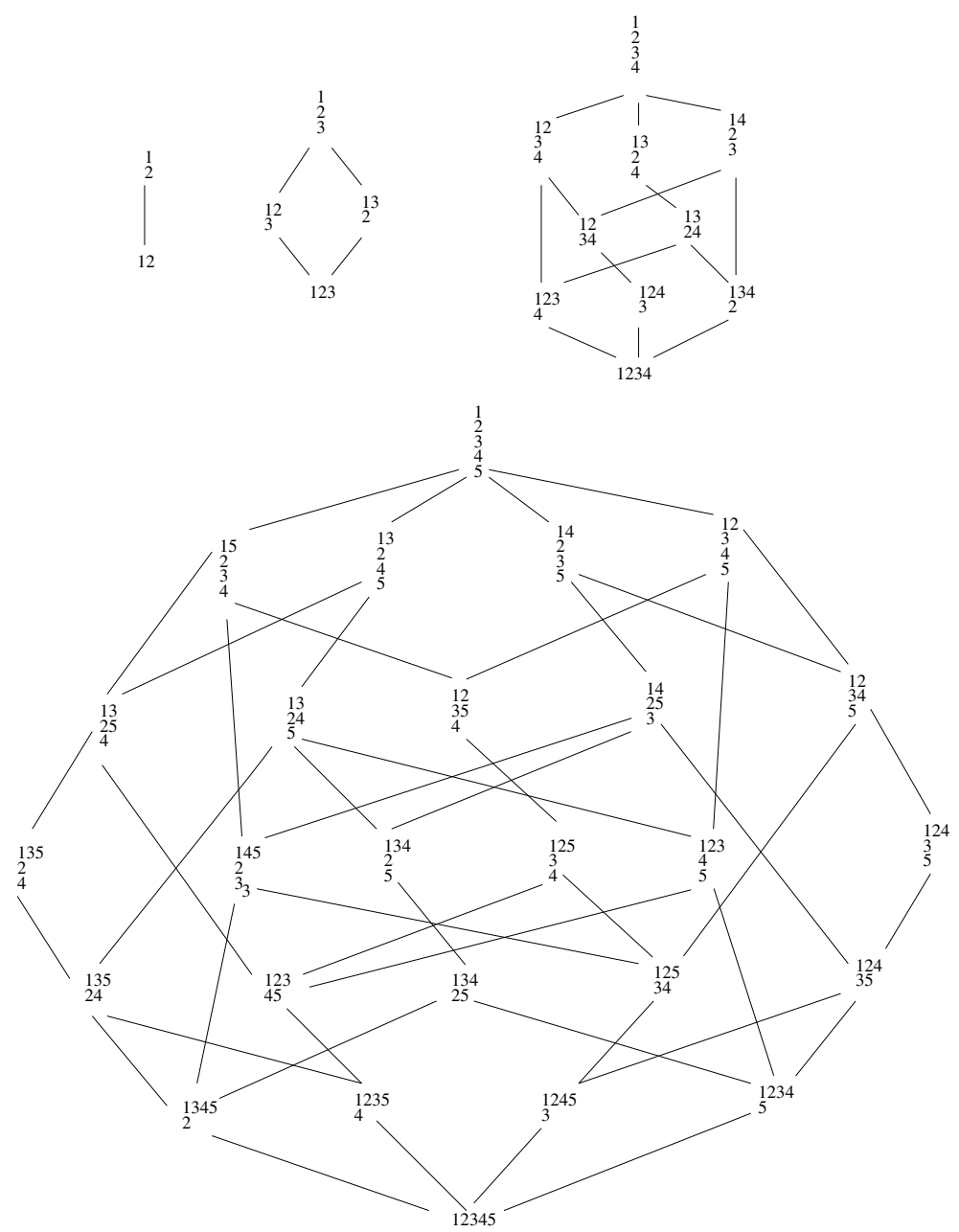

Figure 1. The weak on $S Y T_{n}$ for $n=2,3,4,5$.

Definition 2.9. For $\tau \in S_{n}$ and $T \in S Y T_{n}$ the (left) descent sets are defined as follows:

i) $\operatorname{Des}_{L}(\tau):=\left\{i: 1 \leq i \leq n-1\right.$ and $\left.\tau^{-1}(i)>\tau^{-1}(i+1)\right\}$.

ii) $\operatorname{Des}(T):=\{i: 1 \leq i \leq n-1$ and $i+1$ appears in a row below $i$ in $T\}$.

As a consequence of well-known properties of $R S K$ we have the following basic fact:

Lemma 2.10. For any $\tau \in \mathcal{Y}_{T}$ we have $\operatorname{Des}_{L}(\tau)=\operatorname{Des}(T)$. Thus the left descent set is constant on Knuth classes.

Let $\left(2^{[n-1]}, \subseteq\right)$ be the Boolean algebra of all subsets of $[n-1]$ ordered by inclusion.

Lemma 2.11. The map $\left(S Y T_{n}, \leq_{\text {weak }}\right) \mapsto\left(2^{[n-1]}, \subseteq\right)$ sending any tableau $T$ to its descent set $\operatorname{Des}(T)$ is order preserving. 
Proof. For $n=2$, the statement follows directly by examination of Figure 11. For $n>2$, Lemma 2.8 together with the fact that

$$
\operatorname{Des}_{L}(T)=\operatorname{Des}_{L}\left(T_{[1, n-1]}\right) \cup \operatorname{Des}_{L}\left(T_{[2, n]}\right)
$$

gives the desired result by induction.

Remark 2.12. Melnikov shows in [15, Page 45] that geometric order also restricts to segments as the weak order (see Lemma 2.8). On the other hand, the same fact about Kazhdan-Lusztig order was first shown by Barbash and Vogan [1 for arbitrary finite Weyl groups (see also work by Lusztig [13]) whereas the generalization to Coxeter groups is due to Geck [6, Corollary 3.4]. Therefore Lemma 2.8 and Lemma 2.11 are also true for these orders.

We denote by $\left(\operatorname{Par}_{n}, \leq_{d o m}^{o p}\right)$ the set of all partitions of $n$ ordered by the opposite (or dual) dominance order, that is, $\lambda \leq_{d o m}^{o p} \mu$ if

$$
\lambda_{1}+\cdots+\lambda_{k} \geq \mu_{1}+\cdots+\mu_{k} \text { for all } k .
$$

Lemma 2.13. Let $\leq$ be any of the weak, KL or geometric orders on $S Y T_{n}$. Then

$$
S \supsetneqq T \Longrightarrow \operatorname{shape}(S) \underset{\text { dom }}{o p} \operatorname{shape}(T) ;
$$

i.e., under these orders the shape of the tableaux is not fixed.

Proof. For KL and geometric orders, this property can be induced from the work of Lusztig [12] and Gerstenhaber [5, Chapter 6] respectively. Now $\leq_{\text {weak }}$ satisfies the hypothesis since it is weaker than $K L$ and geometric orders.

Remark 2.14. In fact a combinatorial proof of a weaker statement

$$
S \leq_{\text {weak }} T \Longrightarrow \operatorname{shape}(S) \leq_{\text {dom }}^{o p} \operatorname{shape}(T)
$$

can be easily deduced from Greene's theorem [7].

Recall that for a standard Young tableau $T, T^{t}$ denotes the transpose of $T$ whereas $T^{e v a c}$ denotes the tableau found by applying the Schützenberger [23] evacuation map on $T$. Moreover

$$
\begin{aligned}
& \text { if } \tau=\tau_{1} \tau_{2} \ldots \tau_{n} \in \mathcal{Y}_{T}, \text { then } \tau^{t}=\tau_{n} \tau_{n-1} \ldots \tau_{1} \in \mathcal{Y}_{T^{t}} \\
& \quad \text { and } \tau^{\text {evac }}=\left(n+1-\tau_{n}\right)\left(n+1-\tau_{n-1}\right) \ldots\left(n+1-\tau_{1}\right) \in \mathcal{Y}_{T^{\text {evac }}} .
\end{aligned}
$$

Proposition 2.15. Suppose $S \leq_{\text {weak }} T$ in $S Y T_{n}$. Then

(1) $S^{\text {evac }} \leq_{\text {weak }} T^{\text {evac }}$.

(2) $T^{t} \leq_{\text {weak }} S^{t}$.

Proof. Let $w_{0}$ be the longest element in $S_{n}$. Then the maps

$$
w \mapsto w_{0} w \text { and } w \mapsto w w_{0}
$$

are clearly anti-automorphism, and hence $w \mapsto w_{0} w w_{0}$ is an automorphism of $\left(S_{n}\right.$, $\left.\leq_{\text {weak }}\right)$. On the other hand $I\left(w w_{0}\right)$ is just the transpose tableau of $I(w)$ [22], whereas $I\left(w_{0} w w_{0}\right)$ is nothing but the evacuation of $I(w)[23]$. 
2.3. Inner tableau translation property. The dual Knuth relations $\underset{K^{*}}{\sim}$ on $S_{n}$ play the main role in the definition of the inner tableau translation property. In its most basic form this relation is defined through the Knuth relations applied on the inverse of permutations. Namely,

$$
\sigma \underset{K^{*}}{\sim} \tau \text { in } S_{n} \text { if and only if } \sigma^{-1} \underset{K}{\sim} \tau^{-1} .
$$

An equivalent definition can be given in the following manner: Let

$$
D_{j}^{i}\left(S_{n}\right):=\left\{\alpha \in S_{n} \mid i \in \operatorname{Des}_{L}(\alpha) \text { but } j \notin \operatorname{Des}_{L}(\alpha)\right\} .
$$

Definition 2.16. The inner translation map $\mathcal{V}_{i+1}^{i}: D_{i+1}^{i}\left(S_{n}\right) \mapsto D_{i}^{i+1}\left(S_{n}\right)\left(\mathcal{V}_{i+1}^{i}\right.$ : $\left.D_{i}^{i+1}\left(S_{n}\right) \mapsto D_{i+1}^{i}\left(S_{n}\right)\right)$ is defined through the following action: If $\sigma \in D_{i+1}^{i}\left(S_{n}\right)$ $\left(\sigma \in D_{i}^{i+1}\left(S_{n}\right)\right)$, then

$$
\begin{aligned}
& \text { either } \sigma_{[i, i+2]}=(i+1) i(i+2) \text { or } \sigma_{[i, i+2]}=(i+1)(i+2) i \\
& \text { (either } \left.\sigma_{[i, i+2]}=(i+2) i(i+1) \text { or } \sigma_{[i, i+2]}=i(i+2)(i+1)\right) \text {. }
\end{aligned}
$$

In both cases $\mathcal{V}_{i+1}^{i}(\sigma)$ (and respectively $\mathcal{V}_{i}^{i+1}(\sigma)$ ) is obtained by interchanging the left- and the rightmost numbers of $\sigma_{[i, i+2]}$ in $\sigma$.

Definition 2.17. We say that $\sigma$ and $\tau$ are dual Knuth equivalent through a single dual Knuth relation determined by the triple $\{i, i+1, i+2\}$ if

$$
\text { either } \tau=\mathcal{V}_{i+1}^{i}(\sigma) \text { or } \tau=\mathcal{V}_{i}^{i+1}(\sigma) \text {. }
$$

Moreover we say that $\sigma$ and $\tau$ are dual Knuth equivalent, $\sigma \underset{K^{*}}{\sim} \tau$, if one can be obtained from the other by a sequence of dual Knuth relations.

Following the construction above, now let

$$
D_{j}^{i}\left(S Y T_{n}\right):=\left\{S \in S Y T_{n} \mid i \in \operatorname{Des}(S) \text { but } j \notin \operatorname{Des}(S)\right\} .
$$

Definition 2.18. The inner translation map $\mathcal{V}_{i+1}^{i}: D_{i+1}^{i}\left(S Y T_{n}\right) \mapsto D_{i}^{i+1}\left(S Y T_{n}\right)$ $\left(\mathcal{V}_{i}^{i+1}: D_{i}^{i+1}\left(S Y T_{n}\right) \mapsto D_{i+1}^{i}\left(S Y T_{n}\right)\right)$ is defined through the following action: If $S \in D_{i+1}^{i}\left(S Y T_{n}\right)\left(S \in D_{i}^{i+1}\left(S Y T_{n}\right)\right)$, then we have the following possibilities for $S:$

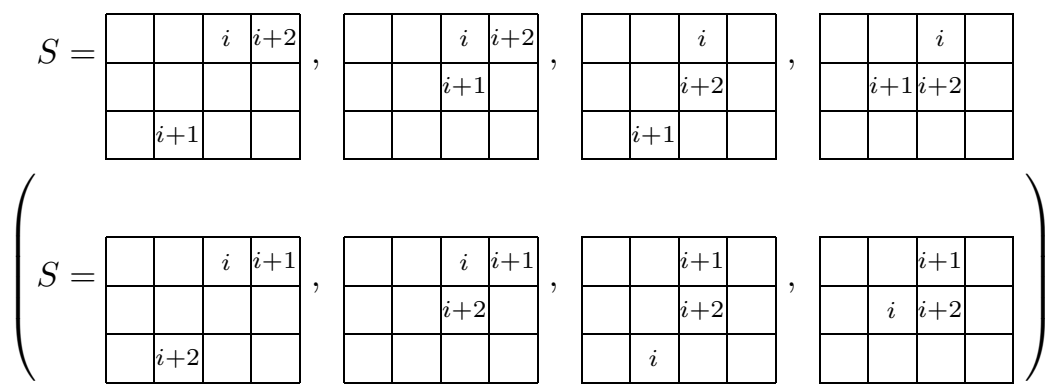

Consider the smallest rectangle containing $\{i, i+1, i+2\}$. Then the southwest and the northeast corners of this rectangle are labeled by either $\{i, i+1\}$ or $\{i+1, i+2\}$. Then $\mathcal{V}_{i+1}^{i}(S)$ is obtained by interchanging the labels of these corners.

Definition 2.19. We say that $S$ and $T$ are dual Knuth equivalent through a single dual Knuth relation determined by the triple $\{i, i+1, i+2\}$ if

$$
\text { either } T=\mathcal{V}_{i+1}^{i}(S) \text { or } T=\mathcal{V}_{i}^{i+1}(S) \text {. }
$$


Moreover we say that $S$ and $T$ are dual Knuth equivalent, $S \underset{K^{*}}{\sim} T$, if one can be obtained from the other by a sequence of dual Knuth relations.

Recall that by Lemma 2.10, we have

$$
\sigma \in D_{j}^{i}\left(S_{n}\right) \text { if and only if } I(\sigma) \in D_{j}^{i}\left(S Y T_{n}\right) .
$$

In fact more is true:

Lemma 2.20. If $\sigma \in D_{i \pm 1}^{i}\left(S_{n}\right)$, then $I\left(\mathcal{V}_{i \pm 1}^{i}(\sigma)\right)=\mathcal{V}_{i \pm 1}^{i}(I(\sigma))$.

Proof. We will just consider the case where $\sigma \in D_{i+1}^{i}\left(S_{n}\right)$ since the other case can be dealt with in the same manner. Now $\sigma$ has the following form: $\sigma=\sigma_{1} \ldots i+$ $1 \ldots i+2 \ldots i \ldots \sigma_{n-1} \sigma_{n}$. First assume that $\sigma_{n}=i$, i.e.,

$$
\sigma=\sigma_{1} \ldots i+1 \ldots i+2 \ldots \sigma_{n-1} i \text { and } \mathcal{V}_{i+1}^{i}(\sigma)=\sigma_{1} \ldots i \ldots i+2 \ldots \sigma_{n-1} i+1 \text {. }
$$

Let $S=I(\sigma), \tilde{S}=I\left(\mathcal{V}_{i+1}^{i}(\sigma)\right), R=I\left(\sigma_{1} \ldots i+1 \ldots i+2 \ldots \sigma_{n-1}\right)$ and $\tilde{R}=$ $I\left(\sigma_{1} \ldots i \ldots i+2 \ldots \sigma_{n-1}\right)$. Clearly

$$
S=I(\sigma)=R^{\downarrow i} \text { and } \tilde{S}=I\left(\mathcal{V}_{i+1}^{i}(\sigma)\right)=\tilde{R}^{\downarrow i+1} .
$$

It is easy to observe that $R$ and $\tilde{R}$ differ by only the label of the same cell, say $C$, which is labeled by $i+1$ in $R$ and by $i$ in $\tilde{R}$ respectively. If $C$ is located in the first row, then $i+2$ must also be in the first row of both $R$ and $\tilde{R}$. Now the insertion of $i$ into $R$ bumps $i+1$ into the next row and the insertion of $i+1$ into $\tilde{R}$ bumps $i+2$ into the next row as illustrated below.

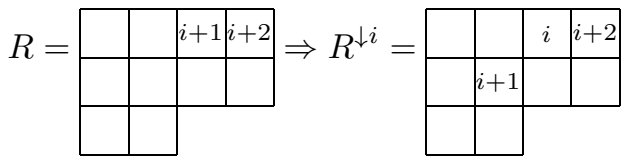

and

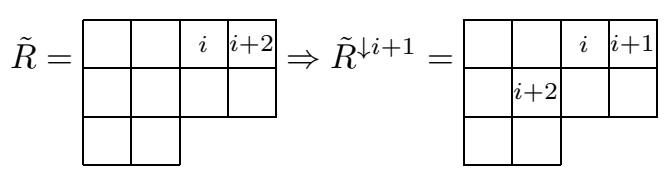

On the other hand, the insertion of $i+1$ and $i+2$ into the second row of the corresponding tableaux follows the same path of cells, since the lower rows of $R$ and $\tilde{R}$ are the same and $i+1$ and $i+2$ are adjacent numbers. Therefore $\tilde{R}^{\downarrow i+1}$ is obtained from $R^{\downarrow i}$ by just interchanging the labels $i+1$ and $i+2$, and thus $\tilde{S}=\mathcal{V}_{i+1}^{i}(S)$, as required.

Now if $C$ is not in the first row, then either $i+1$ and $i+2$ are in the same row or they are in different rows of $R$. We have the following illustrations for $R$ and $\tilde{R}$ :

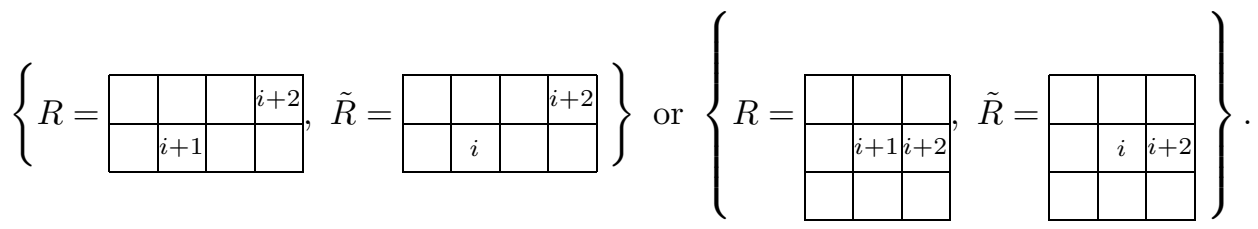

Here it is easy to check that insertion of $i$ into $R$ and $i+1$ into $\tilde{R}$ follows the same path of cells. Thus $S=R^{\downarrow i}$ and $\tilde{S}=\tilde{R}^{\downarrow i+1}$ are obtained from one another by interchanging the position $i+1$ and $i$. So $\tilde{S}=\mathcal{V}_{i+1}^{i}(S)$. 
Now assume that $\sigma_{n} \neq i+1$. Then $\sigma=\ldots i+1 \ldots i+2 \ldots i \ldots \sigma_{n-1} \sigma_{n}$ and $\mathcal{V}_{i+1}^{i}(\sigma)=\ldots i \ldots i+2 \ldots i+1 \ldots \sigma_{n-1} \sigma_{n}$. Let $R=I\left(\ldots i+1 \ldots i+2 \ldots i \ldots \sigma_{n-1}\right)$ and $\tilde{R}=I\left(\ldots i \ldots i+2 \ldots i+1 \ldots \sigma_{n-1}\right)$. By induction we may suppose that

$$
\tilde{R}=\mathcal{V}_{i+1}^{i}(R) .
$$

Since $\tilde{S}=\tilde{R}^{\downarrow \sigma_{n}}$ and $S=R^{\downarrow \sigma_{n}}$, all we need to show is that $\tilde{R}^{\downarrow \sigma_{n}}=\mathcal{V}_{i+1}^{i}\left(R^{\downarrow \sigma_{n}}\right)$. First we suppose that $i, i+1$ and $i+2$ are all located in different rows in $R$ and consider the following illustrations for $R$ and $\tilde{R}$ :

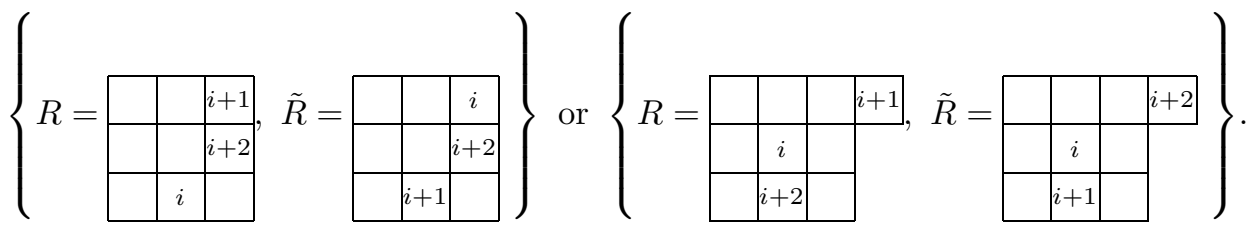

Now in each case the insertion of $\sigma_{n}$ into $R$ and $\tilde{R}$ follows also the same path of cells and hence the resulting tableaux satisfy $\tilde{S}=\mathcal{V}_{i+1}^{i}(S)$.

Otherwise either $i$ and $i+1$ or $i$ and $i+2$ lie in the same row of $R$, as illustrated below:

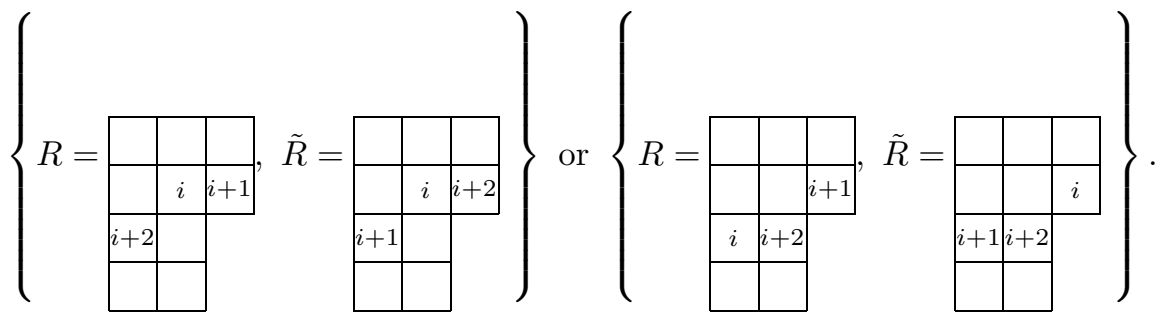

Now in the first case above, the insertion of $\sigma_{n}$ traces the same path of cells in $R$ and $\tilde{R}$, as one can check easily. Thus $\tilde{S}=\mathcal{V}_{i+1}^{i}(S)$, as required. In the second case the only difference in the insertion path of $R^{\downarrow \sigma_{n}}$ and $R^{\downarrow \sigma_{n}}$ can happen if the cell, say $C$, labeled by $i+1$ in $R$ and $i$ in $\tilde{R}$ also belongs to these insertion paths. In this case the label of $C$ in $R(i+1)$ bumps $i+2$ whereas its label in $\tilde{R}(i)$ bumps $i+1$ to the next row. On the other hand, the resulting tableaux still satisfy $\tilde{S}=\mathcal{V}_{i+1}^{i}(S)$ as illustrated below.

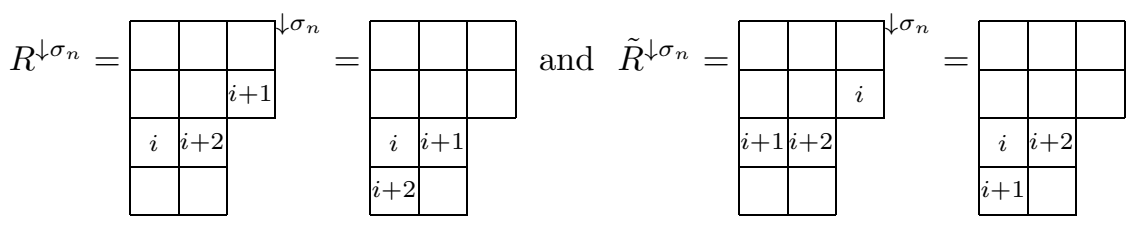

The following result (see [21, Proposition 3.8.1]) has a vital role in our Conjecture 1.1

Proposition 2.21. Let $R, \tilde{R} \in S Y T_{k}$. Then $R \underset{K^{*}}{\sim} \tilde{R}$ if and only if shape $(R)=$ shape $(\tilde{R})$. 
Let $S Y T_{n}^{R}:=\left\{T \in S Y T_{n} \mid T_{[1, k]}=R\right\}$ for some $1 \leq k<n$. Now we define:

Definition 2.22. For $1 \leq k<n$, let $R, \tilde{R} \in S Y T_{k}$ satisfy $\operatorname{shape}(S)=\operatorname{shape}(T)$. Then the inner tableau translation map

$$
\mathcal{V}_{[R, \tilde{R}]}: S Y T_{n}^{R} \mapsto S Y T_{n}^{\tilde{R}}
$$

sends every $T \in S Y T_{n}^{R}$ to the tableau obtained by replacing $R$ with $\tilde{R}$ in $T$.

If a partial order $\left(\leq, S Y T_{n}\right)$ is preserved under the inner tableau translation map, then we say $\left(\leq, S Y T_{n}\right)$ has the inner tableau translation property.

Remark 2.23. As a consequence of Proposition [2.21, one can generate $\mathcal{V}_{[R, \tilde{R}]}(T)$ by a sequence of inner translation maps which act on the subtableau $R$ of $T$. As a result, if a partial order is preserved under the inner translation maps $\mathcal{V}_{i+1}^{i}$ and $\mathcal{V}_{i}^{i+1}$ for each $1 \leq i \leq n-2$, then it is also preserved under the inner tableau translation map.

In fact the inner translation map on $S Y T_{n}$ is first introduced by Vogan in [26], where he also shows that Kazhdan-Lusztig order is preserved under this map. For the geometric order this result is due to Melnikov [18, Proposition 6.6]. Therefore by the remark above, these two orders are also preserved under the inner tableau translation map.

The example given below shows that the weak order is not preserved under the inner translation map. On the other hand, this does not necessitate the same statement for the inner tableau translation map as Conjecture 1.1 asserts.

Example 2.24. One can check that

$$
S=\begin{array}{|l|l|l|}
\hline 1 & 2 & \mathbf{4} \\
\hline \mathbf{3} & \mathbf{5} & 6 \\
\hline
\end{array} \leq_{\text {weak }} T=\begin{array}{|l|l|l|}
\hline 1 & 2 & \mathbf{4} \\
\hline \mathbf{3} & 6 & \multicolumn{1}{|c}{} \\
\cline { 1 - 2 } \mathbf{5} & \multicolumn{1}{|c}{}
\end{array}
$$

but

$$
\mathcal{V}_{3}^{4}(S)=\begin{array}{|l|l|l|}
\hline 1 & 2 & \mathbf{3} \\
\hline \mathbf{4} & \mathbf{5} & 6 \\
\hline
\end{array} \not_{\text {weak }} \mathcal{V}_{3}^{4}(T)=\begin{array}{|l|l|l|}
\hline 1 & 2 & \mathbf{5} \\
\hline \mathbf{3} & 6 & \multicolumn{1}{|c}{} \\
\cline { 1 - 2 } \mathbf{4} & \multicolumn{3}{|l}{}
\end{array}
$$

where the latter pair is obtained from the former by applying a single dual Knuth relation on the triple $\{3,4,5\}$. Here observe that $S_{[1,5]} \neq T_{[1,5]}$. On the other hand $S$ and $T$ below satisfy $S_{[1,4]}=T_{[1,4]}=R$ and

$$
S=\begin{array}{|l|l|l|}
\hline \mathbf{1} & \mathbf{2} & \mathbf{4} \\
\hline \mathbf{3} & 5 & 6 \\
\hline
\end{array} \leq_{\text {weak }} T=\begin{array}{|l|l|l|}
\hline \mathbf{1} & \mathbf{2} & \mathbf{4} \\
\hline \mathbf{3} & 6 & \multicolumn{1}{|l}{} \\
\cline { 1 - 2 } 5 & \multicolumn{2}{|c}{}
\end{array}
$$

and

$$
\mathcal{V}_{[R, \tilde{R}]}(S)=\begin{array}{|l|l|l|}
\hline \mathbf{1} & \mathbf{2} & \mathbf{3} \\
\hline \mathbf{4} & 5 & 6 \\
\hline
\end{array} \leq_{\text {weak }} \mathcal{V}_{[R, \tilde{R}]}(T)=\begin{array}{|l|l|l|}
\mathbf{1} & \mathbf{2} & \mathbf{3} \\
\hline \mathbf{4} & 6 & \multicolumn{1}{|c}{} \\
\cline { 1 - 2 } 5 & \multicolumn{2}{|l}{}
\end{array}
$$

where $R=$\begin{tabular}{|l|l|l}
\hline 1 & 2 & 4 \\
\hline 3 &
\end{tabular} and $\tilde{R}=$\begin{tabular}{|l|l|l|}
\hline 1 & 2 & 3 \\
\hline 4 & &
\end{tabular} have the same shape. 


\section{Applichtions of the COnjecture}

3.1. Well-definedness of the weak order. As was discussed earlier, the only proof for the well-definedness of the weak order uses the inclusion (1.1). We first provide the following self-contained combinatorial proof of this basic fact by assuming Conjecture 1.1.

Theorem 3.1. The weak order on $S Y T_{n}$ is well defined.

Proof. It is enough to show that if $S \leq_{\text {weak }} T$ and $S \neq T$, then $T \not_{\text {weak }} S$. By Lemma 2.11 we know that

$$
S \leq_{\text {weak }} T \text { implies } \operatorname{Des}(S) \subseteq \operatorname{Des}(T)
$$

and if $\operatorname{Des}(T) \neq \operatorname{Des}(S)$, then this yields $T \not Z_{\text {weak }} S$. Now we suppose that $\operatorname{Des}(T)=\operatorname{Des}(S)$. Let $k$ be the largest integer satisfying

$$
S_{[1, k]}=T_{[1, k]} .
$$

Therefore $k<n$ and since $k$ is the largest integer satisfying $S_{[1, k]}=T_{[1, k]}, S_{[1, k+1]}$ and $T_{[1, k+1]}$ must differ only by the position of the corner cells labeled by $k+1$. Furthermore

by Lemma 2.8 and

$$
S_{[1, k+1]} \lessgtr_{w e a k} T_{[1, k+1]}
$$

$$
\operatorname{shape}\left(T_{[1, k+1]}\right) \lesseqgtr_{\text {dom }} \operatorname{shape}\left(S_{[1, k+1]}\right)
$$

by Remark 2.14. Here observe that the case $\operatorname{shape}\left(T_{[1, k+1]}\right)=\operatorname{shape}\left(S_{[1, k+1]}\right)$ implies $S_{[1, k+1]}=T_{[1, k+1]}$, which contradicts the assumption on $k$.

Let $A=\{(i, j)\}$ and $B=\left\{\left(i^{\prime}, j^{\prime}\right)\right\}$ denote the cells labeled by $k+1$ in $S_{[1, k+1]}$ and $T_{[1, k+1]}$ respectively. Then (3.1) implies that

$$
i<i^{\prime} \text { and } j>j^{\prime} \text {; }
$$

i.e., $B$ lies below $A$ and therefore there exists a corner cell $C=\left(i^{\prime \prime}, j^{\prime \prime}\right)$ of $S_{[1, k]}=$ $T_{[1, k]}$ which satisfies

$$
i \leq i^{\prime \prime}<i^{\prime} \text { and } j>j^{\prime} \geq j^{\prime \prime} .
$$

Let $R$ denote $S_{[1, k]}=T_{[1, k]}$ and let $\tilde{R}$ be another tableau in $S Y T_{k}$ such that $\operatorname{shape}(R)=\operatorname{shape}(\tilde{R})$ and the corner cell $C$ of $\tilde{R}$ is labeled by $k$. Denote also by $\tilde{S}$ and $\tilde{T}$ the tableaux obtained by replacing $R$ with $\tilde{R}$ in $S$ and $T$ respectively. Observe that

$$
k \in \operatorname{Des}(\tilde{T})-\operatorname{Des}(\tilde{S}) .
$$

Now by Conjecture 1.1 we have $\tilde{S} \leq_{\text {weak }} \tilde{T}$ and moreover since $\operatorname{Des}(\tilde{T}) \neq \operatorname{Des}(\tilde{S})$, $\tilde{T} \not_{\text {weak }} \tilde{S}$. Once more Conjecture 1.1 implies $T \not_{\text {weak }} S$.

3.2. Poirier-Reutenauer Hopf algebra on $\mathbb{Z} S Y T=\bigoplus_{n>0} \mathbb{Z} S Y T_{n}$. Following the work of Malvenuto and Reutenauer on permutations [14, Poirier and Reutenauer construct two graded Hopf algebra structures on the $\mathbb{Z}$-module of all plactic classes $\left\{P C_{T}\right\}_{T \in S Y T}$, where $P C_{T}=\sum_{P(u)=T} u$. The product structure of the one that concerns us here is given by

$$
P C_{T} * P C_{T^{\prime}}=\sum_{\substack{P(u)=T \\ P(w)=T^{\prime}}} \operatorname{shf}(u, \bar{w}),
$$


where $\bar{w}$ is obtained by increasing the indices of $w$ by the length of $u$ and shf denotes the shuffle product. Then the bijection sending each plactic class to its defining tableau gives us a Hopf algebra structure on the $\mathbb{Z}$-module of all standard Young tableaux, $\mathbb{Z} S Y T=\bigoplus_{n>0} \mathbb{Z} S Y T_{n}$. For detailed explanations, see 4 .

In [19, Poirier and Reutenauer explain this product using jeu-de-taquin slides. Following an analogous result of Loday and Ronco [11, Thm. 4.1] on permutations, the author shows the following result in 25]: For $S \in S Y T_{k}, T \in S Y T_{l}$ where $k+l=n$, let $\bar{T}$ be the tableau obtained by increasing the indices of $T$ by $k$. Denote by $S / T$ the tableau whose columns are obtained by concatenating the columns of $\bar{T}$ over $S$ from below and by $S \backslash T$ the tableau whose rows are obtained by concatenating the rows of $\bar{T}$ over $S$ from the right. Then by [25, Thm. 4.2],

$$
S * T=\sum_{\substack{R \in S Y T_{n}: \\ S \backslash T \leq \text { weak } \\ R \leq \text { weak } S / T}} R .
$$

Namely the product structure can be read on the weak order poset of standard Young tableaux.

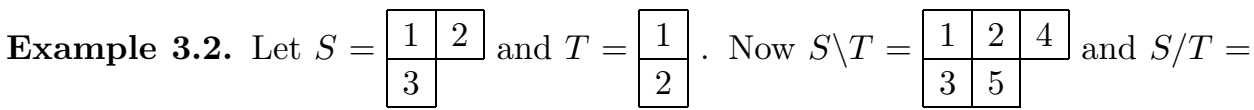
\begin{tabular}{|l|l|l|l|l|l|l}
\hline 1 & 2 & Then \\
\cline { 1 - 1 } 3 &
\end{tabular}

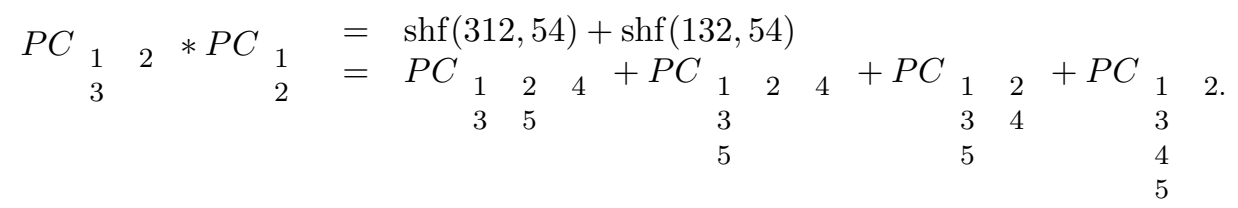

On the other hand, one can check from Figure 1 that the product $S * T$ is equal to the sum of all tableaux in the interval $[S \backslash T, S / T]$.

For $\sigma \in \mathcal{Y}_{S}$ and $\tau \in \mathcal{Y}_{T}$ one can easily check that $\sigma \bar{\tau} \in \mathcal{Y}_{S \backslash T}$ and $\bar{\tau} \sigma \in \mathcal{Y}_{S / T}$. On the other hand,

$$
(\sigma \bar{\tau})^{e v a c}=\tau^{e v a c} \overline{\sigma^{e v a c}} \text { and }(\bar{\tau} \sigma)^{e v a c}=\overline{\sigma^{e v a c}} \tau^{e v a c}
$$

and therefore by (2.2) $(S \backslash T)^{e v a c}=T^{e v a c} \backslash S^{e v a c}$ and $(S / T)^{e v a c}=T^{e v a c} / S^{e v a c}$.

Now as an application of Conjecture 1.1 we have the following result.

Corollary 3.3. Let $S, S^{\prime}, T, T^{\prime}$ be standard Young tableaux satisfying

$$
\operatorname{shape}(S)=\operatorname{shape}\left(S^{\prime}\right) \text { and } \operatorname{shape}(T)=\operatorname{shape}\left(T^{\prime}\right) \text {. }
$$

Then the intervals of the weak order $[S \backslash T, S / T]$ and $\left[S^{\prime} \backslash T^{\prime}, S^{\prime} / T^{\prime}\right]$ are isomorphic. Equivalently, the shuffle product $S * T$ is determined by the shapes of the tableaux rather than the tableaux itself.

Proof. Conjecture 1.1 and Proposition 2.15 yield that

$$
\begin{aligned}
{[S \backslash T, S / T] \cong\left[S^{\prime} \backslash T, S^{\prime} / T\right] } & \cong\left[\left(S^{\prime} \backslash T\right)^{\text {evac }},\left(S^{\prime} / T\right)^{\text {evac }}\right] \\
& =\left[T^{\text {evac }} \backslash\left(S^{\prime}\right)^{\text {evac }}, T^{\text {evac }} /\left(S^{\prime}\right)^{\text {evac }}\right] .
\end{aligned}
$$


On the other hand,

$$
\begin{aligned}
{\left[T^{e v a c} \backslash\left(S^{\prime}\right)^{e v a c}, T^{e v a c} /\left(S^{\prime}\right)^{e v a c}\right] } & \cong\left[\left(T^{\prime}\right)^{\text {evac }} \backslash\left(S^{\prime}\right)^{e v a c},\left(T^{\prime}\right)^{e v a c} /\left(S^{\prime}\right)^{\text {evac }}\right] \\
& \cong\left[S^{\prime} \backslash T^{\prime}, S^{\prime} / T^{\prime}\right]
\end{aligned}
$$

\section{The CASES Where THE CONJECTURE HOLDS}

Lemma 4.1. Suppose $S \lessdot_{\text {weak }} T$ is a covering relation in $S Y T_{n}^{R}$ and $R, \tilde{R} \in S Y T_{k}$ have the same shape. If $\operatorname{std}\left(S_{[k+1, n]}\right) \leq_{\text {weak }} \operatorname{std}\left(T_{[k+1, n]}\right)$, then $\mathcal{V}_{[R, \tilde{R}]}(S) \leq_{\text {weak }}$ $\mathcal{V}_{[R, \tilde{R}]}(T)$.

Proof. It is enough to consider the case when $R$ and $\tilde{R}$ differ only by one dual Knuth relation determined by the triples $\{i, i+1, i+2\}$, for some $i \leq k-2$. Since $S \lessdot_{\text {weak }} T$ is a covering relation, there exist $\sigma \in \mathcal{Y}_{S}$ and $\tau \in \mathcal{Y}_{T}$ such that $\sigma \lessdot \tau$ is also a covering relation for the right weak order on $S_{n}$; i.e., for some $i<n$,

$$
\sigma=a_{1} \ldots a_{j} a_{j+1} \ldots a_{n} \leq a_{1} \ldots a_{j+1} a_{j} \ldots a_{n}=\tau, \text { and } a_{j}<a_{j+1} .
$$

Now by Lemma 2.8 we have

$$
I\left(\sigma_{[k+1, n]}\right)=S_{[k+1, n]} \text { and } T_{[k+1, n]}=I\left(\tau_{[k+1, n]}\right)
$$

and the assumption $S_{[k+1, n]} \lesseqgtr$ weak $T_{[k+1, n]}$ yields that $\sigma_{[k+1, n]} \lesseqgtr \tau_{[k+1, n]}$ and $\sigma_{[1, k]}=\tau_{[1, k]}$.

Therefore applying the dual Knuth relation determined by the triple $\{i, i+1, i+2\}$ on $\sigma$ and $\tau$ gives two new permutations, say $\tilde{\sigma}$ and $\tilde{\tau}$, such that $\tilde{\sigma} \lessdot \tilde{\tau}$ in the right weak Bruhat order, so

$$
\mathcal{V}_{[R, \tilde{R}]}(S)=I(\tilde{\sigma}) \leq_{\text {weak }} I(\tilde{\tau})=\mathcal{V}_{[R, \tilde{R}]}(T) .
$$

Lemma 4.2. Suppose

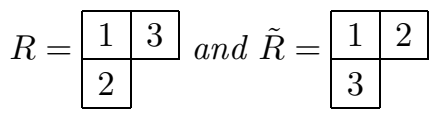

Then $S \lessdot_{\text {weak }} T$ in $S Y T_{n}^{R}$ if and only if $\mathcal{V}_{[R, \tilde{R}]}(S) \lessdot_{\text {weak }} \mathcal{V}_{[R, \tilde{R}]}(T)$ in $S Y T_{n}^{\tilde{R}}$.

Proof. Since $S \lessdot_{\text {weak }} T$ in $S Y T_{n}^{R}$ there exist $\sigma \in \mathcal{Y}_{S}$ and $\tau \in \mathcal{Y}_{T}$ such that for some $1 \leq j<n$ we have

$$
\sigma=a_{1} \ldots a_{j} a_{j+1} \ldots a_{n} \text { and } \tau=a_{1} \ldots a_{j+1} a_{j} \ldots a_{n}, \text { where } a_{j}<a_{j+1}
$$

and $\left\{\sigma_{[1,3]}, \tau_{[1,3]}\right\} \subset \mathcal{Y}_{R}=\{213,231\}$.

If $\left\{a_{j}, a_{j+1}\right\} \neq\{1,3\}$, then applying the dual Knuth relation determined by $\{1,2,3\}$ on $\sigma$ and $\tau$ yields two permutations $\tilde{\sigma} \in \mathcal{V}_{[R, \tilde{R}]}(S)$ and $\tilde{\tau} \in \mathcal{V}_{[R, \tilde{R}]}(T)$ which still have $\tilde{\sigma} \lessdot \tilde{\tau}$ in the right weak order. Therefore $\mathcal{V}_{[R, \tilde{R}]}(S) \leq_{\text {weak }} \mathcal{V}_{[R, \tilde{R}]}(T)$.

Suppose $\left\{a_{j}, a_{j+1}\right\}=\{1,3\}$. Then since $\mathcal{Y}_{R}=\{213,231\}$, the number 2 is the smallest number in $a_{1} \ldots a_{j-1}$ and it labels the leftmost cell in the first of $I\left(a_{1} \ldots a_{j-1}\right)$. Let

$$
b_{1} \ldots b_{i-1} 2 b_{i+1} \ldots b_{j-1}
$$

be the row word of $I\left(a_{1} \ldots a_{j-1}\right)$ obtained by reading numbers in each row from left to right, starting from the last row. Therefore

$$
a_{1} \ldots a_{j-1} \underset{K}{\sim} b_{1} \ldots b_{i-1} 2 b_{i+1} \ldots b_{j-1}
$$


and hence

$$
\begin{aligned}
& \sigma^{\prime}=b_{1} \ldots b_{i-1} 2 b_{i+1} b_{i+2} \ldots b_{j-1} 13 a_{j+2} \ldots a_{n} \in \mathcal{Y}_{S} \\
& \tau^{\prime}=b_{1} \ldots b_{i-1} 2 b_{i+1} b_{i+2} \ldots b_{j-1} 31 a_{j+2} \ldots a_{n} \in \mathcal{Y}_{T} .
\end{aligned}
$$

Observe that since the subword $2 b_{i+1} \ldots b_{j-1}$ labels the first row of $I\left(a_{1} \ldots a_{j-1}\right)$ we have

$$
2<b_{i+1}<\ldots<b_{j-1}
$$

and therefore

$$
\begin{aligned}
& \sigma^{\prime} \underset{K}{\sim} \sigma^{\prime \prime}=b_{1} \ldots b_{i-1} 2 b_{i+1} 13 b_{i+2} \ldots b_{j-1} a_{j+2} \ldots a_{n}, \\
& \tau^{\prime} \underset{K}{\sim} \tau^{\prime \prime}=b_{1} \ldots b_{i-1} b_{i+1} 213 b_{i+2} \ldots b_{j-1} a_{j+2} \ldots a_{n} .
\end{aligned}
$$

Hence we get other permutations $\sigma^{\prime \prime} \in \mathcal{Y}_{S}$ and $\tau^{\prime \prime} \in \mathcal{Y}_{T}$ satisfying $\sigma^{\prime \prime} \lessdot \tau^{\prime \prime}$ in the right weak Bruhat order. Now applying single dual Knuth relations determined by $\{1,2,3\}$ on these permutations gives respectively two permutations $\tilde{\sigma} \in \mathcal{Y}_{\mathcal{V}_{[R, \tilde{R}]}(S)}$ and $\tilde{\tau} \in \mathcal{Y}_{\mathcal{V}_{[R, \tilde{R}]}(T)}$ which still satisfy $\tilde{\sigma} \lessdot \tilde{\tau}$ in the right weak Bruhat order. Therefore $\mathcal{V}_{[R, \tilde{R}]}(S) \leq_{\text {weak }} \mathcal{V}_{[R, \tilde{R}]}(T)$.

Now let $\tilde{S}=\mathcal{V}_{[R, \tilde{R}]}(S)$ and $\tilde{T}=\mathcal{V}_{[R, \tilde{R}]}(T)$ and $\tilde{S} \lessdot_{\text {weak }} \tilde{T}$ be a covering relation in $S Y T_{n}^{\tilde{R}}$. By Proposition 2.15 we have

$$
\tilde{T}^{t} \lessdot_{\text {weak }} \tilde{S}^{t} \text { in } S Y T_{n}^{R} \text {. }
$$

Since $\tilde{R}^{t}=R$, now by the previous result we have $\mathcal{V}_{[R, \tilde{R}]}\left(\tilde{T}^{t}\right) \leq_{\text {weak }} \mathcal{V}_{[R, \tilde{R}]}\left(\tilde{S}^{t}\right)$ and so

$$
\left(\mathcal{V}_{[R, \tilde{R}]}\left(\tilde{S}^{t}\right)\right)^{t} \leq_{\text {weak }}\left(\mathcal{V}_{[R, \tilde{R}]}\left(\tilde{T}^{t}\right)\right)^{t} .
$$

On the other hand observe that $S=\left(\mathcal{V}_{[R, \tilde{R}]}\left(S^{t}\right)\right)^{t}$ and $T=\left(\mathcal{V}_{[R, \tilde{R}]}\left(T^{t}\right)\right)^{t}$ and hence $S \leq_{\text {weak }} T$ in $S Y T_{n}^{R}$.

Now suppose $S \lessdot_{\text {weak }} T$ in $S Y T_{n}^{R}$ but $\mathcal{V}_{[R, \tilde{R}]}(S) \leq_{\text {weak }} \mathcal{V}_{[R, \tilde{R}]}(T)$ is not a covering relation in $S Y T_{n}^{\tilde{R}}$. Then there exists a tableau $Q \in S Y T_{n}^{\tilde{R}}$ satisfying $\mathcal{V}_{[R, \tilde{R}]}(S) \lessgtr_{\text {weak }} Q \lessgtr_{\text {weak }} \mathcal{V}_{[R, \tilde{R}]}(T)$, but this implies

$$
S \lesseqgtr_{\text {weak }} \mathcal{V}_{[\tilde{R}, R]}(Q) \lessgtr_{\text {weak }} T
$$

which is clearly a contradiction. Hence $\mathcal{V}_{[R, \tilde{R}]}(S) \lessdot_{\text {weak }} \mathcal{V}_{[R, \tilde{R}]}(T)$.

Proposition 4.3. Suppose that $S \lessdot_{\text {weak }} T$ in $S Y T_{n}^{R}$, where $R \in S Y T_{k}$ has exactly two rows. If $\tilde{R}$ is another tableau in $S Y T_{k}$ having the same shape as $R$, then $\mathcal{V}_{[R, \tilde{R}]}(S) \lessdot_{w e a k} \mathcal{V}_{[R, \tilde{R}]}(T)$ in $S Y T_{n}^{\tilde{R}}$.

Proof. Suppose that $S \lessdot_{\text {weak }} T$ in $S Y T_{n}^{R}$ and $R \in S Y T_{k}$ has two rows. When $k<3$ there is nothing to prove since $R$ can be only vertical and horizontal. For $k=3$ the only case that needs to be explored is when shape $(R)=(2,1)$, and Lemma 4.2 gives the desired result.

So we suppose the statement is true for $k-1$ and let $R \in S Y T_{k}$. It is enough to consider the case when $R$ and $\tilde{R}$ differ by only one dual Knuth relation determined by the triple $\{i, i+1, i+2\}$ where $i+2 \leq k$. If $i+2<k$, then the subtableau 
$S_{[1, i+2]}=T_{[1, i+2]}$ of $R$ still has two rows, and by induction we have the desired result. If $i+2=k$, then we have the following possibilities for $R$ :

\begin{tabular}{|c|c|c|c|}
\hline$*$ & $*$ & $i$ & $i+2$ \\
\hline$*$ & $*$ & $i+1$ & \multicolumn{1}{|c}{} \\
\cline { 1 - 2 } & &
\end{tabular}

(a)

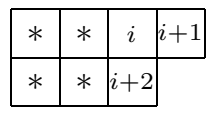

(b)

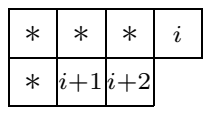

(c)

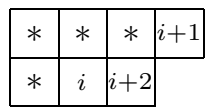

$(d)$

Observe that in the last two classes the dual Knuth relation determined by $\{i$, $i+1, i+2\}$ interchanges the places of $i+1$ and $i$, and so one can apply the induction hypothesis on the smaller inner tableau $S_{[1, i+1]}=T_{[1, i+1]}$ to get the required result.

For the first two classes we have the following analysis: Since $S \lessdot_{\text {weak }} T$ there exist $\sigma \in \mathcal{Y}_{S}$ and $\tau \in \mathcal{Y}_{T}$ such that $\sigma<\tau$ is also a covering relation, the right weak order on $S_{n}$; i.e., for some $1 \leq j<n$ we have

$$
\sigma=a_{1} \ldots a_{j} a_{j+1} \ldots a_{n} \text { and } \tau=a_{1} \ldots a_{j+1} a_{j} \ldots a_{n}, \text { where } a_{j}<a_{j+1} .
$$

If $\left\{a_{j}, a_{j+1}\right\} \neq\{i, i+2\}$, then applying the dual Knuth relation determined by $\{i, i+1, i+2\}$ on $\sigma$ and $\tau$ yields two permutations $\tilde{\sigma} \in \mathcal{V}_{[R, \tilde{R}]}(S)$ and $\tilde{\tau} \in \mathcal{V}_{[R, \tilde{R}]}(T)$, which still have $\tilde{\sigma} \lessdot \tilde{\tau}$ in the right weak order. Therefore $\mathcal{V}_{[R, \tilde{R}]}(S) \leq_{\text {weak }} \mathcal{V}_{[R, \tilde{R}]}(T)$.

Now let $\left\{a_{j}, a_{j+1}\right\}=\{i, i+2\}$, i.e.,

$$
\sigma=a_{1} \ldots a_{j-1} i(i+2) a_{j+2} \ldots a_{n} \text { and } \tau=a_{1} \ldots a_{j-1}(i+2) i a_{j+2} \ldots a_{n} .
$$

Case 1. We first consider the case where the inner tableau $R$ has the form illustrated in (4.1)-(a). Here since $i+1$ does not belong to the descent sets of $S$ and $T, i+1$ must come before $i+2$ in every permutation in the Knuth classes of $S$ and $T$. Therefore $i+1$ must lie in $a_{1} \ldots a_{j-1}$ and it must be located either on the first row or the second row of $I\left(a_{1} \ldots a_{j-1}\right)$. Now observe that if $i+1$ is on the second row, then inserting $(i+2) i$ in $I\left(a_{1} \ldots a_{j-1}\right)$ results in a tableau having $i+2$ on its second row, but this clearly contradicts the assumption on $R$. Therefore $i+1$ must lie in the first row of $I\left(a_{1} \ldots a_{j-1}\right)$. Let

$$
b_{1} \ldots b_{r-1}(i+1) b_{r+1} b_{r+2} \ldots b_{j-1}
$$

be the row word of $I\left(a_{1} \ldots a_{j-1}\right)$ obtained by reading numbers in each row from left to right, starting from the last row. Now since

$$
a_{1} \ldots a_{j-1} \underset{K}{\sim} b_{1} \ldots b_{r-1}(i+1) b_{r+1} b_{r+2} \ldots b_{j-1}
$$

this yields that

$$
\begin{gathered}
\sigma \underset{K}{\sim} \sigma^{\prime}=b_{1} \ldots b_{r-1}(i+1) b_{r+1} b_{r+2} \ldots b_{j-1} i(i+2) a_{j+2} \ldots a_{n}, \\
\tau \underset{K}{\sim} \tau^{\prime}=b_{1} \ldots b_{r-1}(i+1) b_{r+1} b_{r+2} \ldots b_{j-1}(i+2) i a_{j+2} \ldots a_{n} .
\end{gathered}
$$

On the other hand, since $i+1$ lies in the first row, we have $i+1<b_{r+1}<b_{r+2}<$ $\ldots<b_{j-1}$, and therefore

$$
\begin{aligned}
\sigma^{\prime} \underset{K}{\sim} \sigma^{\prime \prime} & =b_{1} \ldots b_{r-1}(i+1) b_{r+1} i(i+2) b_{i+2} \ldots b_{j-1} a_{j+2} \ldots a_{n}, \\
\tau^{\prime} \underset{K}{\sim} \tau^{\prime \prime} & =b_{1} \ldots b_{r-1} b_{r+1}(i+1) i(i+2) b_{r+2} \ldots b_{j-1} a_{j+2} \ldots a_{n} .
\end{aligned}
$$

Now applying single dual Knuth relations determined by $\{i, i+1, i+2\}$ on $\sigma^{\prime \prime}$ and $\tau^{\prime \prime}$ gives respectively two permutations $\tilde{\sigma} \in \mathcal{Y}_{\mathcal{V}_{[R, \tilde{R}]}(S)}$ and $\tilde{\tau} \in \mathcal{Y}_{\mathcal{V}_{[, \tilde{R}]}(T)}$ which still satisfy $\tilde{\sigma} \lessdot \tilde{\tau}$ in the right weak Bruhat order. Hence $\mathcal{V}_{[R, \tilde{R}]}(S) \leq_{\text {weak }} \mathcal{V}_{[R, \tilde{R}]}(T)$. 
Case 2. Now we consider the case illustrated in (4.1)-(b). Here by Proposition 2.15,

$$
T^{t} \leq_{\text {weak }} S^{t} \in S Y T_{n}^{R^{t}}
$$

where $R^{t}$ is a tableau having at most two columns. Moreover the permutations

$$
\sigma^{t}=a_{n} \ldots(i+1) \ldots a_{j+2} i+2(i) a_{j-1} \ldots a_{1}
$$

and

$$
\tau^{t}=a_{n} \ldots(i+1) \ldots a_{j+1}(i) i+2 a_{j-1} \ldots a_{1}
$$

which are obtained by reversing $\sigma$ and $\tau$ of (4.2) lie in $\mathcal{Y}_{S^{t}}$ and $\mathcal{Y}_{T^{t}}$ respectively.

Now consider the tableau $I\left(a_{n} \ldots(i+1) \ldots a_{j+2}\right)$. Suppose first that the leftmost cell in its first row is labeled by a number, say $x$. Now if $x<i+1$, then the insertion of $i(i+2)$ into $I\left(a_{n} \ldots(i+1) \ldots a_{j+2}\right)$ places $i(i+2)$ to the right of $x$, but this contradicts the fact that the inner tableau $R^{t}$ has at most two columns. Therefore $x=i+1$; i.e., $i+1$ lies in the first row of $I\left(a_{n} \ldots(i+1) \ldots a_{j+2}\right)$, as in Case 1 . Moreover it labels the leftmost cell in this row. After this point one can apply the same arguments used in Case 1 to show that

$$
\tilde{T}^{t} \leq_{\text {weak }} \tilde{S}^{t} \in S Y T_{n}^{\tilde{R}^{t}}
$$

and hence $\tilde{S} \leq_{\text {weak }} \tilde{T} \in S Y T_{n}^{\tilde{R}}$, as required.

Lastly, the fact that resulting relations are in fact covering relations follows directly.

Corollary 4.4. Suppose that $S \lessdot_{\text {weak }} T$ in $S Y T_{n}^{R}$, where $R \in S Y T_{k}$ has exactly two columns. If $\tilde{R} \in S Y T_{k}$ has the same shape as $R$, then $\mathcal{V}_{[R, \tilde{R}]}(S) \lessdot_{\text {weak }} \mathcal{V}_{[R, \tilde{R}]}(T)$ in $S Y T_{n}^{\tilde{R}}$.

Proof. By Proposition 2.15 we have $T^{t} \lessdot_{w e a k} S^{t}$ in $S Y T_{n}^{R^{t}}$, where $R^{t}$ has exactly two rows. Now $(\tilde{R})^{t}$ has the same shape with $R^{t}$ and by the previous theorem $\mathcal{V}_{\left[R^{t},(\tilde{R})^{t}\right]}\left(T^{t}\right) \lessdot_{\text {weak }} \mathcal{V}_{\left[R^{t},(\tilde{R})^{t}\right]}\left(S^{t}\right)$. Therefore

$$
\mathcal{V}_{[R, \tilde{R}]}(S)=\left(\mathcal{V}_{\left[R^{t}, \tilde{R}^{t}\right]}\left(S^{t}\right)\right)^{t} \lessdot_{w e a k}\left(\mathcal{V}_{\left[R^{t}, \tilde{R}^{t}\right]}\left(T^{t}\right)\right)^{t}=\mathcal{V}_{[R, \tilde{R}]}(T)
$$

Definition 4.5. Let $T \in S Y T_{n}$ and $A$ be a corner cell of shape(T). Then

i) $T^{\uparrow A}$ denotes the tableau obtained by applying the reverse insertion algorithm to $T$ through the corner cell $A$;

ii) $\eta\left(T^{\uparrow A}\right)$ denotes the number which leaves the tableau at the end of the reverse insertion $T^{\uparrow A}$.

The following result on the hook shape tableaux is easy to deduce by using the reverse RSK algorithm.

Lemma 4.6. Let $R \in S Y T_{k}$ be a tableau of a hook shape with more then two rows and two columns and suppose that the only two corner cells of $R$, say $A$ and $B$, are labeled by $k$ or $k-1$. Then

$$
\eta\left(R^{\uparrow A}\right) \neq \eta\left(R^{\uparrow B}\right)
$$

and if $a_{1} \ldots a_{k}$ and $b_{1} \ldots b_{k}$ are two permutations in the Knuth class of $R$ with $a_{k}=b_{k}$, then

$$
I\left(a_{1} \ldots a_{k-1}\right)=I\left(b_{1} \ldots b_{k-1}\right) .
$$


Proof. Since the tableaux are required to have more then two rows and two columns, it is enough to consider the following tableaux, where $k$ labels the cell $A$ and $k-1$ labels the cell $B$ :
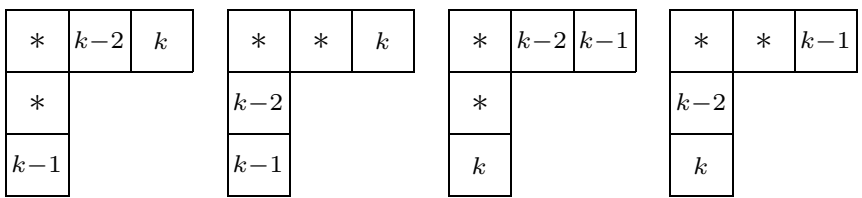

Clearly $\eta\left(R^{\uparrow A}\right) \neq \eta\left(R^{\uparrow B}\right)$ and this shows that if $a_{k}=b_{k}$, then they must leave the tableau at the end of a reverse insertion applied on the same corner cell, say $A$. Therefore $I\left(a_{1} \ldots a_{k-1}\right)=R^{\uparrow A}=I\left(b_{1} \ldots b_{k-1}\right)$.

Proposition 4.7. Suppose that $S \lessdot_{\text {weak }} T$ in $S Y T_{n}^{R}$, where $R \in S Y T_{k}$ has a hook shape. If $Q$ is another tableau in $S Y T_{k}$ having the same shape with $R$, then $\mathcal{V}_{[R, Q]}(S) \lessdot_{\text {weak }} \mathcal{V}_{[R, Q]}(T)$ in $S Y T_{n}^{Q}$.

Proof. When $R$ is a horizontal or vertical tableau there is nothing to prove. On the other hand for $k \leq 4$, a nonhorizontal or nonvertical tableau has either two rows or two columns, and so Proposition 4.3 gives the required result. In what follows, we assume that $n>k>4$ and $R$ has more than two rows and two columns.

It is enough to consider the case that $R$ and $Q$ differ by only one dual Knuth relation determined by the triple $\{i, i+1, i+2\}$. If $i+2<k$, then the subtableau $S_{[1, i+2]}=T_{[1, i+2]}$ of $R$ still has a hook shape and induction gives the desired result. So let $i+2=k$; i.e., $R$ and $Q$ differ by a single dual Knuth relation determined by $\{k-2, k-1, k\}$. Observe that since $R$ has a hook shape, the dual Knuth relation interchanges the places of $k$ and $k-1$. Thus the only two corners of $R$ are labeled by $k$ and $k-1$.

Now since $S \lessdot_{\text {weak }} T$ is a covering relation, there exist $\sigma \in \mathcal{Y}_{S}$ and $\tau \in \mathcal{Y}_{T}$ such that $\sigma \lessdot \tau$ in the right weak Bruhat order on $S_{n}$; i.e., for some $1 \leq j<n$ we have

$$
\sigma=a_{1} \ldots a_{j} a_{j+1} \ldots a_{n} \text { and } \tau=a_{1} \ldots a_{j+1} a_{j} \ldots a_{n}, \text { where } a_{j}<a_{j+1} .
$$

If $\left\{a_{j}, a_{j+1}\right\} \neq\{k, k-2\}$, then as discussed earlier, the result follows as in the proof of Proposition 4.3. So in the remainder we assume that $\left\{a_{j}, a_{j+1}\right\}=\{k, k-2\}$.

Observe that we have either $\sigma_{1}=a_{1}=\tau_{1}$ or $\sigma_{n}=a_{n}=\tau_{n}$. W.L.O.G. assume $\sigma_{n}=a_{n}=\tau_{n}$ (the other case can be dealt with in the same manner after taking the transposes of the tableaux). Therefore there exist some corner cells, say $A_{S}$ and $A_{T}$ of $S$ and $T$ respectively, such that

$$
a_{n}=\eta\left(S^{\uparrow A_{S}}\right)=\eta\left(T^{\uparrow A_{T}}\right),
$$

and let $S^{\prime}=I\left(a_{1} \ldots a_{j} a_{j+1} \ldots a_{n-1}\right)=S^{\uparrow A_{S}}$ and $T^{\prime}=I\left(a_{1} \ldots a_{j+1} a_{j} \ldots a_{n-1}\right)=$ $T^{\uparrow A_{T}}$. We still have

$$
S^{\prime} \leq_{\text {weak }} T^{\prime}
$$

Case 1. If $a_{n}>k$, then $S^{\prime}$ and $T^{\prime}$ still have the same inner tableau $R$. Moreover since $S^{\prime} \leq_{\text {weak }} T^{\prime}$, we have by induction

$$
\mathcal{V}_{[R, Q]}\left(S^{\prime}\right) \leq_{\text {weak }} \mathcal{V}_{[R, Q]}\left(T^{\prime}\right)
$$

and therefore $\mathcal{V}_{[R, Q]}(S)=\mathcal{V}_{[R, Q]}\left(S^{\prime}\right)^{\downarrow a_{n}} \leq_{\text {weak }} \mathcal{V}_{[R, Q]}\left(T^{\prime}\right)^{\downarrow a_{n}}=\mathcal{V}_{[R, Q]}(T)$. 
Case 2. If $a_{n} \leq k$, then the fact that $a_{n}$ belongs to $R$ implies that in both reverse insertions $S^{\uparrow A_{S}}$ and $T^{\uparrow A_{T}}$ the number $a_{n}$ leaves both tableaux through some path which goes through $R$. Therefore for some corners $C$ and $C^{\prime}$ of $R$, we have

$$
a_{n}=\eta\left(R^{\uparrow C}\right)=\eta\left(R^{\uparrow C^{\prime}}\right) .
$$

Recall that the only two corners of $R$ are labeled by $k$ and $k-1$ and that $R$ has more than two rows and two columns. Now by Lemma 4.6] it follows that $C=C^{\prime}$ and $C$ is labeled by either $k$ or $k-1$.

First suppose that $k$ is in the first row of $R$ (hence of $S$ and $T$ ) as illustrated below.

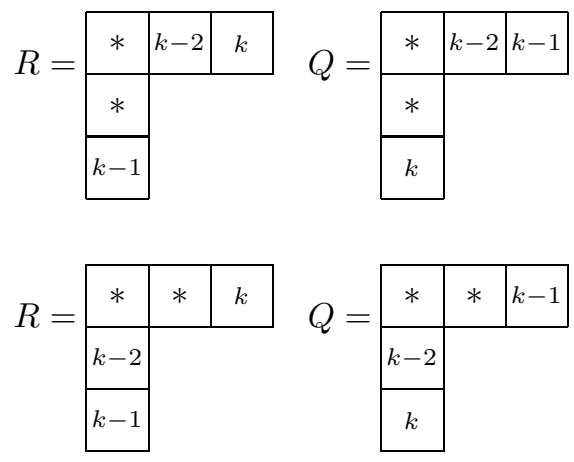

Then, since $a_{n}=\eta\left(R^{\uparrow C}\right)=\eta\left(S^{\uparrow A_{S}}\right)=\eta\left(T^{\uparrow A_{T}}\right)$ we have either $a_{n}=k$ or $a_{n} \leq k$. Now if $a_{n}=k$, then the corner cell $C$ of $R$ must be the cell labeled by $k$. On the other hand, this case gives a contradiction since $\left\{a_{j}, a_{j+1}\right\}=\{k, k-2\}$. If $a_{n} \leq k-2$, then the corner cell $C$ of $R$ must be the cell labeled by $k-1$, and the reverse insertion $R^{\uparrow C}$ in both cases bumps a number which is strictly less than $k-2$. Now let

$$
R^{\prime}=R^{\uparrow C} .
$$

Then one can still apply the dual Knuth relation determined by $\{k-2, k-1, k\}$ to the inner tableau $R^{\prime}$ and let $Q^{\prime}$ be the resulting tableau. Then it can be easily observed from the illustrations above that

$$
Q^{\prime}=Q^{\uparrow C}
$$

Recall that $S^{\prime}=S^{\uparrow A_{S}}$ and $T^{\prime}=T^{\uparrow A_{T}}$ are two tableaux having the same inner tableau $R^{\prime}=R^{\uparrow C}$ and $S^{\prime} \leq_{\text {weak }} T^{\prime}$. Now by induction

$$
\mathcal{V}_{\left[R^{\prime}, Q^{\prime}\right]}\left(S^{\prime}\right) \leq_{\text {weak }} \mathcal{V}_{\left[R^{\prime}, Q^{\prime}\right]}\left(T^{\prime}\right)
$$

and therefore $\mathcal{V}_{[R, Q]}(S)=\mathcal{V}_{\left[R^{\prime}, Q^{\prime}\right]}\left(S^{\prime}\right)^{\downarrow a_{n}} \leq_{\text {weak }} \mathcal{V}_{\left[R^{\prime}, Q^{\prime}\right]}\left(T^{\prime}\right)^{\downarrow a_{n}}=\mathcal{V}_{[R, Q]}(T)$.

Now suppose that $k-1$ is in the first row of $R$ (hence of $S$ and $T$ ) as illustrated below.

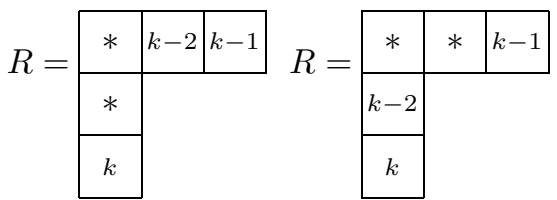

Then either $a_{n}=k-1$ or $a_{n}<k-2$. If $a_{n}<k-2$, then one can apply the same method to get the required result. On the other hand, if $a_{n}=k-1$, then the tableau $R^{\prime}=R^{\uparrow C}$ is obtained by erasing the corner cell of $R$, labeled by $k-1$ above. Then 
clearly every permutation in corresponding Knuth classes has the same subsequence $k(k-2)$. This shows that any two permutations in the Knuth class of $R$ that end with $k-1$ must have the same subsequence $k(k-2)$ and this again contradicts the assumption $\left\{a_{j}, a_{j+1}\right\}=\{k, k-2\}$ in (4.4).

Lastly, the fact that resulting relations are in fact covering relations follows directly.

\section{REFERENCES}

1. D. Barbash, D. Vogan, Primitive ideals and orbital integrals in complex exceptional groups, J. Algebra, 80 (1983), 350-382. MR691809 (84h:22038)

2. A. Björner, Topological Methods, Handbook of Combinatorics (R. Graham, M. Gröschel and L. Lovász, eds.), Elsevier, Amsterdam (1995), 1819-1872. MR.1373690 (96m:52012)

3. A. Björner, M. L. Wachs, Permutation statistics and linear extensions of posets, J. Combin. Theory Ser. A, 58, no. 1 (1991), 85-114. MR.1119703(92m:06010)

4. D. Blessenohl, M. Schocker, Noncommutative Character Theory of the Symmetric Group, Imperial College Press, London (2005). MR2424338 (2009i:20021)

5. D. H. Collingwood, W. M. McGovern, Nilpotent orbits in semisimple Lie algebras, Van Nostrand Reinhold, New York (1993). MR1251060 (94j:17001)

6. M. Geck, On the induction of Kazhdan-Lusztig cells, Bull. London Math. Soc., 35 (2003), 608-614. MR1989489(2004d:20003)

7. C. Greene, An extension of Schensted's theorem, Adv. in Math., 14 (1974), 254-265. MR0354395(50:6874)

8. D. Kazhdan, G. Lusztig, Representations of Coxeter groups and Hecke algebras, Invent. Math., 53 (1979), 165-184. MR560412 (81j:20066)

9. D. E. Knuth, The art of computer programming, sorting and searching, Vol. 3, AddisonWesley (1973), 49-72. MR0445948 (56:4281)

10. D. E. Knuth, Permutations, matrices and generalized Young tableaux, Pacific J. Math., 34 (1970), 709-727. MR0272654 (42:7535)

11. J. Loday, M. Ronco, Order structure on the algebra of permutations and of planar binary trees, J. Algebraic Combin., 15 (2002), 253-270. MR.1900627 (2003m:05213)

12. G. Lusztig, Cells in affine Weyl groups. II, J. of Algebra, 109 (1987), 536-548. MR.902967 $(88 \mathrm{~m}: 20103 \mathrm{a})$

13. G. Lusztig, Characters of reductive groups over a finite field, Ann. Math. Stud. 107, Princeton University Press (1984). MR742472 (86j:20038)

14. C. Malvenuto, C. Reutenauer, Duality between quasi-symmetric functions and the Solomon descent algebra, J. Algebra, 177 (1995), 967-982. MR.1358493 (97d:05277)

15. A. Melnikov, On orbital variety closures in $\mathfrak{s l}_{n}$, I. Induced Duflo order, J. Algebra, 271 (2004), 179-233. MR2022481 (2004m:17009)

16. A. Melnikov, On orbital variety closures in $\mathfrak{s l}_{n}$, II. Descendants of a Richardson orbital variety, J. Algebra, 271 (2004), 698-724. MR.2025547 (2004k:17013)

17. A. Melnikov, Irreducibility of the associated varieties of simple highest modules in $\mathfrak{s l}_{n}$, C.R. Acad. Sci. Paris Ser. I Math., 316 (1993), 53-57. MR1198749 (93k:17012)

18. A. Melnikov, On orbital variety closures in $\mathfrak{s l}_{n}$, III. Geometric properties, J. Algebra, 305 (2006), 68-97. MR2262520(2007j:17011)

19. S. Poirier, C. Reutenauer, Algèbres de Hopf de tableaux, Ann. Sci. Math. Québec, 19 (1995), no. 1, 79-90. MR.1334836 (96g:05146)

20. A. Reifegerste, Permutation sign under the Robinson-Schensted correspondence, Ann. Comb., 8 (2004), no. 1, 103-112. MR2061380 (2005a:05214)

21. B. E. Sagan, The Symmetric Group, Second edition. Springer-Verlag, New York (2001). MR1824028 (2001m:05261)

22. C. Schensted, Longest increasing and decreasing subsequences, Canad. J. Math., 13 (1961), 179-191. MR0121305 (22:12047)

23. M. P. Schützenberger, Quelques remarques sur une construction de Schensted, Math. Scand., 12 (1963), 117-128. MR0190017 (32:7433) 
24. M. P. Schützenberger, La correspondence de Robinson, Combinatoire et Représentation du Groupe Symétrique, Lecture Notes in Math., 579, Springer, Berlin (1977), 59-135. MR0498826 (58:16863)

25. M. Taskin, Properties of four partial orders on standard Young tableaux, J. of Combin. Theory Ser. A., 113 (2006), 1092-1119. MR.2244136(2007h:05162)

26. D. Vogan Jr., Ordering of the primitive spectrum of a semisimple Lie algebra, Math. Ann. 248 (1980), 195-2003. MR.575938 (81k:17006)

Department of Mathematics, Bỡaziçi Üniversitesi, 34342 Bebek-İstanbul, TürkiYe

E-mail address: muge.taskin@boun.edu.tr 